19 Revue d'histoire du XIXe siècle

Société d'histoire de la révolution de 1848 et des

révolutions du XIXe siècle

$39 \mid 2009$

Le monde de l'imprimé: des territoires aux acteurs -

Education et politique - Histoires politiques

\title{
L'espace parisien des libraires sous la monarchie de Juillet : des solidarités de métier ?
}

Booksellers network within Paris during the July Monarchy: an actual solidarity?

Viera Rebolledo-Dhuin

\section{OpenEdition}

Journals

Édition électronique

URL : http://journals.openedition.org/rh19/3914

DOI : $10.4000 /$ rh 19.3914

ISSN : $1777-5329$

Éditeur

La Société de 1848

Édition imprimée

Date de publication : 10 décembre 2009

Pagination : 37-57

ISSN : 1265-1354

Référence électronique

Viera Rebolledo-Dhuin, « L'espace parisien des libraires sous la monarchie de Juillet : des solidarités de métier ? », Revue d'histoire du XIXe siècle [En ligne], 39 | 2009, mis en ligne le 15 décembre 2012, consulté le 03 mai 2019. URL : http://journals.openedition.org/rh19/3914; DOI : 10.4000/rh19.3914 


\section{VIERA REBOLLEDO-DHUIN}

\section{L'espace parisien des libraires sous la monarchie de Juillet: des solidarités de métier ${ }^{1}$ ?}

"D'ailleurs, ça ne me regarde pas, moi, les malheurs de mes proches, reprit Bidault-Gigonnet. J'ai pour principe de ne jamais me laisser aller ni avec mes amis, ni avec mes parents, car on ne peut périr que par les endroits faibles.»

Balzac, Les Employés, Folio, Paris, Gallimard, 1985, p. 198.

Bidault, dit Gigonnet, que les lecteurs de la Comédie Humaine connaissent comme un investisseur averti, fait ici preuve d'une froideur propre aux manieurs d'argent. Cependant, Gigonnet revient rapidement sur ses propos et n'hésite pas à s'engager avec ses confrères Gobseck, Chaboisseau et Métivier - véritable clique - auprès de Des Lupeaulx, afin de soutenir sa nièce, et plus précisément le mari de celle-ci, Baudoyer, contre l'ennemi Rabourdin, grâce à des opérations financières complexes. Ce Gigonnet n'est pourtant pas un banquier expérimenté, mais un simple papetier qui se convertit à l'escompte.

Le présent article tente justement de montrer que l'escompte des Gens du livre et, plus précisément, les réseaux de crédit des libraires en faillite constituent une des clefs de compréhension de l'espace parisien des libraires, un des angles d'approche des continuités et discontinuités socio-spatiales de l'inscription économique et sociale d'un métier entre 1830 et 1848.

La première difficulté que soulève cette étude provient des sources et plus particulièrement des dénominations professionnelles utilisées dans les différents corpus. En effet, le terme générique de libraire désigne tout à la fois le bookseller et le publisher et ce, au moins jusqu'aux années 1850, date à laquelle l'éditeur et le libraire se séparent. Dans l'Almanach du Commerce de Didot Bottin, la double dénomination d' "éditeur-libraire" n'opère pas de distinction : près de 1400 individus sont ainsi recensés entre 1825 et 1845 .

1. Cet article poursuit la réflexion engagée lors de notre communication au colloque international de l'Université de Rome 3, Descriptio urbis. Measuring and Comparating the modern and contemporary city, 28 mars 2008. 
Cependant la dénomination choisie par les acteurs devient parfois problématique et le terme d'éditeur peut répondre à une stratégie d'évitement des obligations législatives. En effet, tout comme l'imprimerie, la librairie - mais non l'édition, qui n'existe pas en tant que telle dans la première moitié du siè$\mathrm{cle}^{2}$ - est soumise, entre 1810 et 1870, au régime du brevet. En dehors d'une copie d'acte de naissance, les dossiers de demande de brevet sont constitués de certificats attestant l'honorabilité du postulant et d'enquêtes réalisées dans le voisinage par le préfet de police. Cette source, inépuisable pour l'historien du livre, rend compte tant du parcours que de l'ensemble des acteurs entourant le futur libraire et permet une analyse diachronique de l'inscription sociale et géographique des candidats à la librairie. Il ne s'agit pourtant pas ici de faire un examen systématique des 700 dossiers de libraires brevetés sous la monarchie de Juillet, mais de ne retenir que quelques dossiers d'individus sur lesquels davantage d'informations peuvent être obtenues en croisant les différents corpus de sources, notamment celui des faillites.

La librairie parisienne connaît en effet au cours de la monarchie de Juillet une profonde mutation, qui se manifeste par une série de faillites, culminant entre 1826 et 1831, avec des prolongements jusqu'aux lendemains des révolutions de 1848 . Au delà de la conjoncture morose, cette multiplication des faillites révèle des réseaux complexes de crédit. Les dossiers de faillite contiennent un nombre variable de pièces dont les plus intéressantes sont, ici, les listes de créanciers vérifiés par le juge du Tribunal de Commerce et les rapports de syndics. Ces pièces, parce qu'elles évaluent la solvabilité - sinon l'honnêteté - du débiteur, rendent compte de la réputation de ce dernier ${ }^{3}$.

L'analyse des listes de créanciers, véritable instantané à un moment financier critique, croisée à celle, en positif et sur la longue durée, des Bottins du Commerce et des dossiers de brevet, permet d'appréhender le milieu de la librairie dans son ensemble. Cette approche se démarque, par deux aspects, des traditions qui caractérisent l'historiographie du livre. D'une part, l'histoire du livre est largement dominée par les études monographiques ${ }^{4}$ qui, pour des raisons évidentes de sources disponibles, se concentrent sur ce qu'Élias Regnault nomme les «barons de l'édition» ou les «Dieux du commerce $»^{5}$, et où dossiers de brevet et de faillite ne constituent qu'un événement

2. Les éditeurs existent et s'affirment sous la monarchie de Juillet : Jean-Yves Mollier, «La police de la librairie. 1810-1881", dans Patricia Sorel et Frédérique Leblanc [dir.], Histoire de la Librairie, Paris, Cercle de la Librairie, 2008, p. 16-26; Christine S. Haynes, Lost Illusions. The Rise of the Book publisher and the construction of a Literary market place in Nineteenth Century France, Ph. D, University of Chicago, Dept. of History, 2001. Mais, ce n'est qu'en 1852 que les éditeurs sont clairement soumis aux mêmes obligations que les libraires.

3. Natacha Coquery et Nicolas Praquin, «Règlement des faillites et pratiques judiciaires : de l'entre soi à l'expertise du syndic (1673-1889)", Histoire et mesure, 2008, vol. 23, n 1, p. 43-83.

4. Jean-Yves Mollier, L'histoire de l'édition, du livre et de la lecture en France de la fin du XVIIIe siècle au début du XXI siècle : approche bibliographique, bibliographie mise à jour en septembre 2008, En Ligne, CHCS, http://www.chcsc.uvsq.fr/semin/BIBLIOGRAPHIEHistoireeditionseptembre2008.pdf

5. Élias Regnault, "L'éditeur", dans Les Français peints par eux-mêmes. Encyclopédie morale du XIXe siècle, Paris, Léon Curmer, 1840-1842, 10 volumes, volume 4 (1841), p. 322-334. 
ponctuel au sein d'un long parcours réussi, sinon prometteur. Au contraire, le présent article tente de redonner une réalité aux petits libraires - pour la plupart, restés dans l'ombre - à partir notamment des dossiers de faillite, afin de comprendre un secteur dans son ensemble, avec la totalité de ses acteurs ${ }^{6}$. D'autre part, en dehors de quelques études économiques ${ }^{7}$, les recherches sur le livre adoptent le plus souvent une approche culturelle, où la question de l'inscription géographique reste secondaire. Or, il s'agit ici de comprendre les enjeux de l'inscription spatiale de la librairie à Paris, tout à la fois pour mieux appréhender l'espace vécu au quotidien par une catégorie professionnelle et analyser le rôle de l'espace dans l'exercice de ce commerce. L'objectif est donc de voir en quoi l'espace physique est simultanément support et révélateur d'un espace social et économique. Cela suppose de s'interroger sur plusieurs objets de natures différentes - le quartier, les réseaux de crédit, la faillite pour déterminer si l'intensité et la structure sociale - c'est-à-dire la sociabilité au premier sens du terme - de cet espace physique et professionnel entrâ̂nent ou non des relations sociales amicales, sinon de solidarité.

Trois aspects peuvent être abordés, en distinguant les différents corpus de sources. Les Bottins du commerce permettent tout d'abord de délimiter l'espace parisien du livre. Les dossiers de brevet ${ }^{8}$ donnent ensuite à voir une représentation, officielle sinon théorique, de l'espace social tout à la fois professionnel et géographique des libraires. Les dossiers de faillites ${ }^{9}$, enfin, rendent visible l'espace social à l'œuvre, l'espace de crédibilité aux deux sens du terme : espace de crédit et de confiance.

\section{Le Quartier latin, «Quartier Général» De la librairie}

Les librairies sont extrêmement concentrées dans l'espace parisien. Plus de deux tiers des boutiques recensées par les Bottins au cours du régime de Juillet se situent dans les $\mathrm{II}^{\mathrm{e}}, \mathrm{X}^{\mathrm{e}}$ et $\mathrm{XI}^{\mathrm{e}}$ arrondissements anciens, et plus particulièrement dans ceux de la rive gauche. En réalité, malgré les évolutions de la géographie parisienne du livre au cours de la monarchie de Juillet, l'ancrage

6. Autour de Frédéric Barbier et Sabine Juratic, ou de Roméo Arbour, des études prosopographiques ont vu le jour, mais pour des périodes ou groupes sociaux limités. Frédéric Barbier, Sabine Juratic et Michel Vangheluwe [dir.], Lumières du Nord. Imprimeurs, libraires et "gens du livre» dans le Nord de la France au XVIII siècle. Dictionnaire prosopographique, Genève, Droz, 2002; Frédéric Barbier, Sabine Juratic et Fabienne Mellerio [dir.], Dictionnaire des imprimeurs, libraires et gens du livre à Paris (17011789), Genève, Droz, 2007; Roméo Arbour, Dictionnaire des femmes libraires en France (1470-1870), Genève, Droz, 2003.

7. Jean-Yves Mollier, L'Argent et les lettres. Histoire du capitalisme d'édition. 1880-1920, Paris, Fayard, 1988 ; Frédéric Barbier, «L'industrialisation des techniques» ou «La production multipliée», dans Roger Chartier et Henri-Jean Martin [dir.], Histoire de l'édition française, 4 tomes, tome 3, Le temps des éditeurs, du romantisme à la Belle Époque, Paris, Fayard/Éditions du Cercle de la Librairie, 1990 (1 $1^{\text {re }}$ édition 1985), p. 51-91, 105-130.

8. Arch. nat. (Archives nationales), F18.

9. Arch. Paris (Archives de Paris), D11 U3. 
historique dans les quartiers de la rive gauche reste de règle, voire se renforce, le quartier Latin exerçant un véritable monopole dans ce secteur.

Tableau 1 : Répartition des libraires par arrondissement

\begin{tabular}{|c|c|c|c|c|c|c|c|c|c|c|c|c|c|c|c|}
\hline \multirow{2}{*}{ Années } & \multicolumn{10}{|c|}{ Arrondissements parisiens } & \multicolumn{4}{c|}{ Communes } & \multirow{2}{*}{ Ensemble } \\
\cline { 2 - 17 } & I & II & III & IV & V & VI & VII & VIII & IX & X & XI & XII & Batignolles & Grenelle & \\
\hline 1825 & 29 & 70 & 32 & 16 & 9 & 16 & 10 & 11 & 14 & 71 & 179 & 24 & & 1 & 482 \\
\hline 1835 & 24 & 76 & 26 & 29 & 9 & 17 & 9 & 11 & 15 & 68 & 166 & 19 & & 1 & 470 \\
\hline 1845 & 28 & 64 & 24 & 18 & 12 & 21 & 3 & 19 & 12 & 94 & 172 & 14 & 1 & & 482 \\
\hline
\end{tabular}

Carte 1 : Évolution de la répartition des libraires parisiens par arrondissement et par habitant

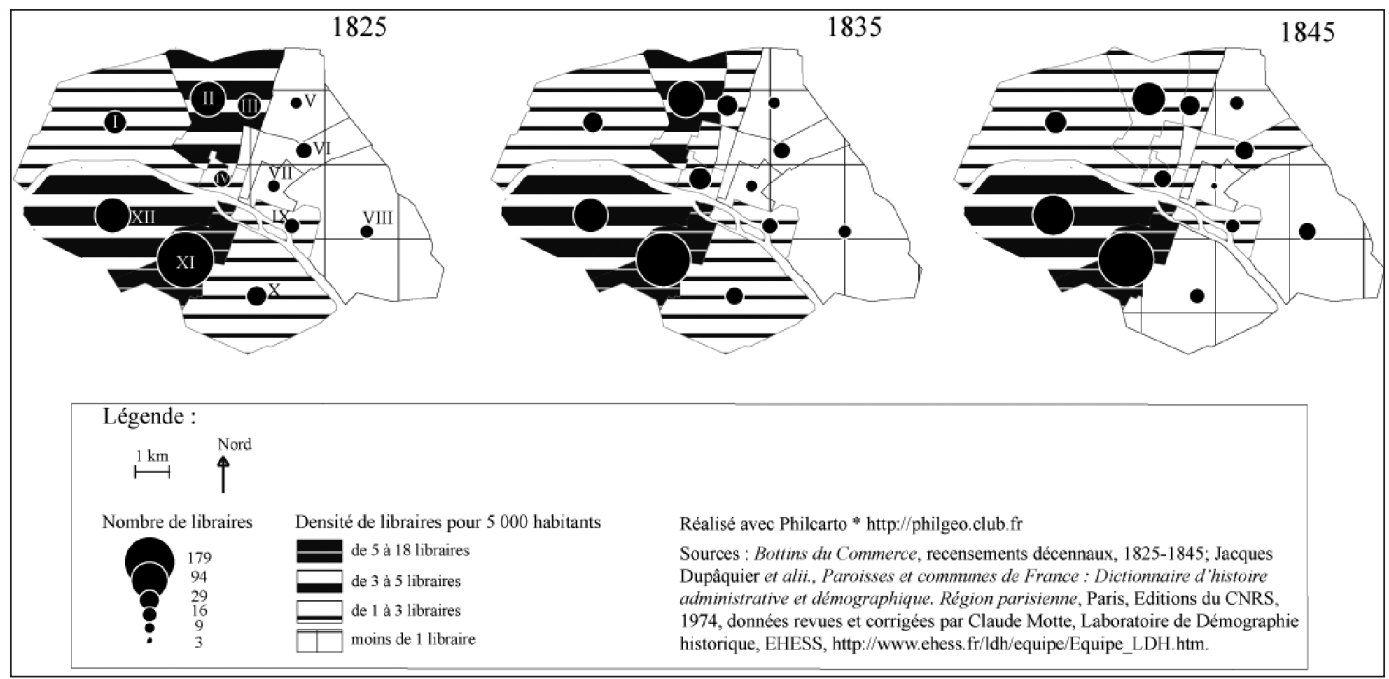

À l'intérieur de ces arrondissements, quatre quartiers dominent, deux à deux limitrophes : rive gauche, les quartiers de l'École de Médecine et de la Monnaie; rive droite, ceux du Palais Royal et Feydeau. Cette localisation est en partie héritée des contraintes pesant sur la librairie d'Ancien Régime : représentants d'un commerce potentiellement séditieux, imprimeurs et libraires sont contraints de travailler dans les quartiers de la Cité, de l'Université ou à proximité de Notre-Dame. Dès la fin du XVIII e siècle, un mouvement s'amorce : les libraires de la rive gauche se déplacent vers l'Ouest de cette même rive et traversent parfois la Seine pour investir le quartier à la mode du Palais Royal ${ }^{10}$. Cette évolution sensible, qui se fait surtout au détriment du

10. Sabine Juratic, "La librairie parisienne des Lumières, 1723-1789», dans Frédéric Barbier [dir.], Paris, capitale des livres. Le monde des livres et de la presse à Paris, du Moyen Âge au XXe siècle, Catalogue d'exposition, Paris, PUF, 2007, p. 191-200. 
$\mathrm{X}^{\mathrm{e}}$ arrondissement, semble pourtant connaître un coup d'arrêt une fois passées les premières années de la monarchie de Juillet. Dès lors, les deux quartiers du quartier Latin regroupent près d'un tiers des librairies parisiennes.

\section{Carte 2 : Évolution de la dispersion des libraires par quartier}

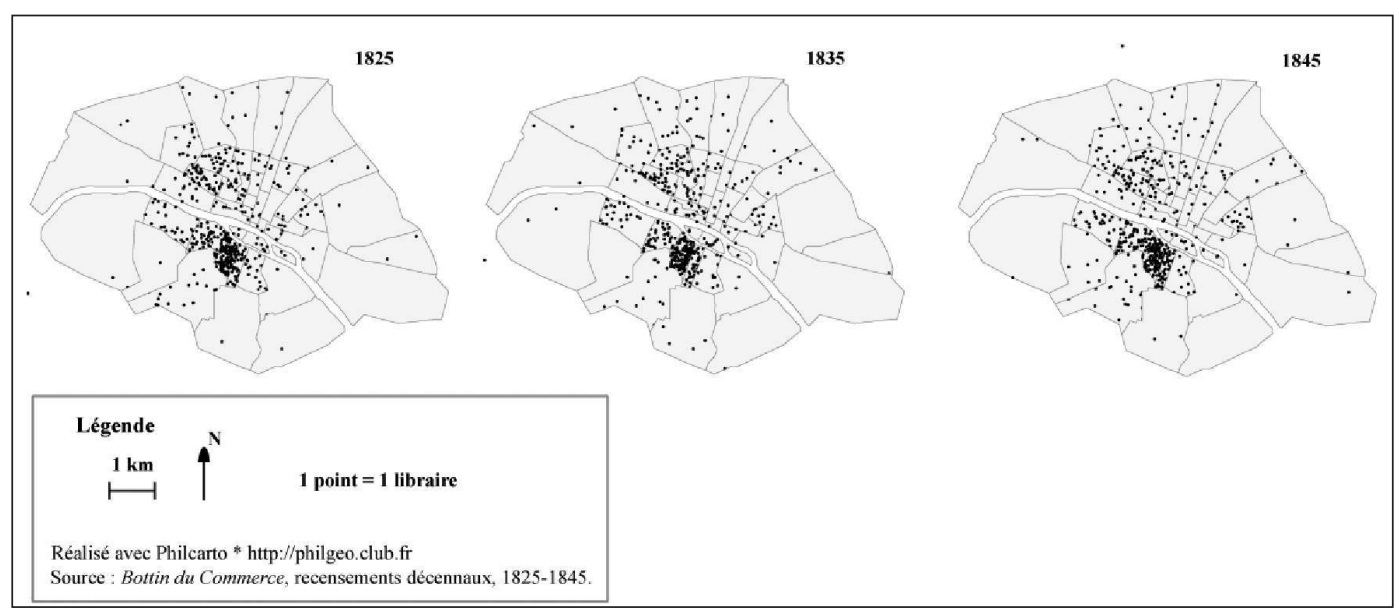

Le caractère relativement éphémère de la librairie du Palais Royal souligne paradoxalement son dynamisme et les spécificités des deux pôles de ce commerce. Situées tout à la fois derrière les Tuileries où viennent flâner les Parisiens aisés, à proximité des boulevards où se développent les scènes de théâtre et à côté de la bourse où se font toutes les affaires, ces boutiques bénéficient d'une conjoncture locale favorable dont elles savent profiter. En effet, le Palais Royal compte un grand nombre de libraires prestigieux parmi lesquels Martin Bossange, libraire du roi, ou le très "fashionable» Ladvocat, modèle utilisé par Balzac pour le portrait de Dauriat ${ }^{11}$. Pour répondre à leur clientèle bourgeoise, les tenants de boutiques à lire se spécialisent dans la vente de livres rares ou religieux, de pièces de théâtre ou de traités d'économie. En outre, pour faire face à la demande croissante et aux besoins de consultation des journaux d'un public affairé, certains tiennent également un cabinet de lecture : près d'un libraire sur cinq détient, à côté de sa boutique, un cabinet littéraire. Cette particularité explique l'importance des femmes libraires, dont certaines célibataires ${ }^{12}$. Au contraire, dans le quartier de la Monnaie, les femmes sont deux fois moins nombreuses tandis que dans celui de l'École de Médecine, si elles ne sont pas absentes, il s'agit essentiellement de veuves

11. Honoré de Balzac, Illusions perdues, Paris, Livre de Poche, 1983 (1 $1^{\text {re }}$ édition 1837-1843). Sur les typologies de libraires antérieures à Balzac : Christine S. Haynes, Lost Illusions..., ouv. cité, p. 78-89.

12. Françoise Parent-Lardeur, Lire à Paris au temps de Balzac. Les cabinets de lecture à Paris, 18151830, Paris, Éditions de l'EHESS, 1999 (1 re édition 1981). 


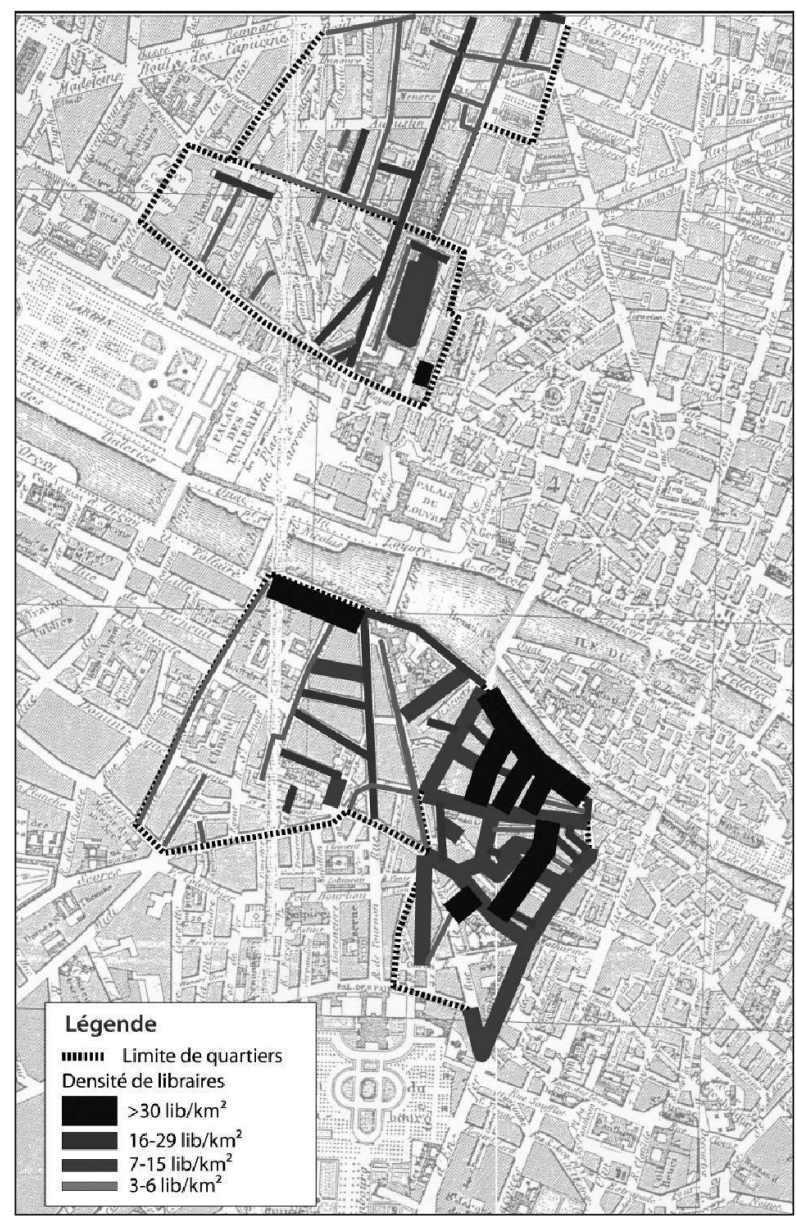

Carte 3 : Densité moyenne de libraires au sein des rues des quatre «quartiers du livre»

reprenant la boutique de leur mari ${ }^{13}$. Cette ouverture propre au quartier de la rive droite n'est pas toujours synonyme de succès puisque dès 1845 , le recensement des Bottins note un repli certain vers les quartiers traditionnels de la rive gauche. Le quartier Latin exerce donc une hégémonie pérenne.

Plus qu'une circonscription administrative, le quartier est un ensemble d'artères, tout à la fois lieux de passage et de jonction ${ }^{14}$. En effet, l'extrême concentration des boutiques de librairie, visible au niveau des quartiers administratifs, est d'autant plus sensible encore lorsque l'on considère l'espace vécu des commerçants. L'espace parisien du livre, organisé autour de quel-

13. Jusqu'en 1791, seules les veuves peuvent tenir une librairie. Malgré l'abolition des corporations, ce monopole masculin perdure de fait tout au long du XIX ${ }^{\mathrm{e}}$ siècle.

14. Alain Cabantous, «Le quartier, espace vécu à l'époque moderne. Ambiguïté et perspectives d'une histoire", Histoire, économie et société, 1994, vol. 13, nº 3, p. 427-439. 
ques rues seulement et faisant fi des délimitations officielles, est discontinu et fortement polarisé.

Sur la rive droite, les galeries du Palais Royal ainsi que les passages à proximité des théâtres fonctionnent comme des centres attractifs pour les rues attenantes qui sont également très prisées. De l'autre côté du fleuve, la concentration est encore plus importante. Les quais et notamment celui des Augustins, où se compte un libraire tous les cinq mètres - voire plusieurs confrères au même numéro -, polarisent les boutiques de librairie. Les rues adjacentes - Gît le Cœur, Pavée, Grands Augustins et plus loin Hautefeuille - bénéficient de cette hégémonie et rayonnent à leur tour sur le "village» Saint-André des Arts et au-delà, rue Dauphine ou rue de la Harpe. Ici les limites administratives ne rendent pas compte de l'espace du livre, délimité au Nord par le quai des Augustins et celui de Malaquais, au Sud par les rues de l'École de Médecine et de l'Abbaye, à l'Est par la rue de la Harpe et à l'Ouest par celle des Petits Augustins, voire par la rue de Seine.

Sans nier la diversité des activités au sein de ces «îlots», ni même la mobilité de quelques libraires - pourtant encore minoritaires ${ }^{15}$-, l'espace de la librairie apparaît structuré autour du quartier entendu comme un ensemble de rues présentant une unité fonctionnelle et une homogénéité professionnelle certaine. Si le quartier constitue une entité topographique et sociale mal délimitée ${ }^{16}$, la proximité d'individus partageant des intérêts quotidiens communs suppose l'existence d'un espace relationnel, perçu et vécu par les acteurs.

\section{L'INSCRIPTION DE LA LIBRAIRIE DANS LE QUARTIER : UNE NÉCESSITÉ}

La concentration spatiale des libraires ne relève pas seulement d'un héritage politique des temps anciens, elle répond également, entre autres ${ }^{17}$, aux contraintes législatives et économiques du premier XIX ${ }^{\mathrm{e}}$ siècle qui imposent des liens de sociabilités inscrits dans le quartier.

Le régime du brevet établi en $1810^{18}$ restaure une logique réglementaire mise à mal lors de la suppression des corporations. Un numerus clausus est

15. Voir carte 4; Marie-Claire Boscq, «L’implantation des libraires à Paris (1815-1848)», dans JeanYves Mollier [dir.], Le Commerce de la librairie en France au XIX'e siècle, 1798-1914, Paris, IMEC/Maison des Sciences de l'Homme, 1997, p. 27-50.

16. Laurent Clavier, " "Quartier" et expériences politiques dans les faubourgs du nord-est parisien en 1848", Revue d'histoire du XIX'e siècle, n 33, 2006/2, p. 124.

17. Françoise Parent-Lardeur, Lire au temps de Balzac..., ouv. cité; Marie-Claire Boscq, «L'implantation des libraires...», art. cité. Gaël Mesnage, «De la rive gauche à la banlieue, l'imprimé dans Paris au XIXe siècle», Histoire et civilisation du livre, vol. 5/2009, à paraître.

18. Bernard Vouillot, «La Révolution et l'Empire; une nouvelle réglementation », dans Roger Chartier et Henri-Jean Martin [dir.], Histoire de l'édition française..., ouv. cité, tome 2, Le livre triomphant,

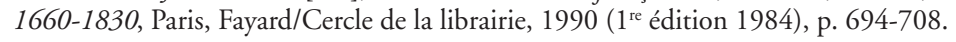


rétabli dans l'imprimerie et l'entrée dans la librairie est contrôlée jusqu'à l'avènement de la Troisième République. Tout demandeur de brevet doit présenter à l'inspecteur de la Librairie, dépendant du ministère de l'Intérieur, un acte de naissance, un certificat de bonne vie et mœurs, un autre de capacités; auxquels s'ajoutent des enquêtes sur le candidat, émanant notamment de la préfecture de police. Plusieurs historiens ${ }^{19}$ ont montré que, de manière générale, les autorités étaient particulièrement sensibles à l'instruction, à la moralité ainsi qu'à la position politique des requérants. Au-delà, l'analyse des pièces constituant les dossiers de brevet met au jour l'importance des relations que doit détenir le libraire pour exister professionnellement, autrement dit l'indispensable construction d'un espace social qui, souvent, recouvre celui lié à la proximité géographique.

Les certificats de capacités, qui doivent être signés par trois professionnels, supposent que le futur libraire connaisse des collègues reconnus, susceptibles d'être crédibles et d'apporter une caution morale. L'obtention du brevet est donc en partie fonction de la renommée des témoins et, de fait, le nombre de signataires varie selon leur réputation, leur crédit. Certains postulants, comme Marie Eugène Belin, justifient l'absence d'un des témoins par la célébrité des signataires ${ }^{20}$. En effet, les imprimeurs dont il s'agit ici, Ernest Panckoucke et Auguste Alexis Pillet aîné, sont largement connus dans le milieu du livre parisien, voire français. Le premier reprend à son compte l'œuvre de son père et de son grand-père : le Moniteur universel. Le second, de moins grand renom - ce qui explique en partie qu'il inscrive sa filiation dans son patronyme - est l'imprimeur de la Bibliographie de la France, de la Revue de Paris ou encore de Flaubert ${ }^{21}$. Certains, au contraire, font preuve de zèle, comme la mère de Marie Eugène Belin, Adèle Mandar qui, en 1834, lorsqu'elle demande le transfert du brevet de son mari à son profit - celui-ci ouvrant une imprimerie à Saint-Cloud ${ }^{22}$ - présente quatre témoins. Elle veut sans doute souligner par là l'ampleur de ses connaissances, mais également pallier l'absence de relations prestigieuses ${ }^{23}$, relations que la famille BelinMandar finit par obtenir à la génération suivante ${ }^{24}$.

Au contraire, les certificats de moralité, dits de bonne vie et mœurs, mon-

19. Pierre Casselle, "Le régime législatif», dans Roger Chartier et Henri-Jean Martin [dir.], Histoire de l'édition française, ouv. cité, tome 3, p. 42-50; Nicole Felkay, Balzac et ses éditeurs, 1822-1837. Essai sur la librairie romantique, Paris, Promodis/Cercle de la Librairie, 1987, p. 25-31.

20. Arch. nat., F18 1732.

21. Odile Krakovitch, Les imprimeurs parisiens sous Napoléon $1^{e r}$. Édition critique de l'enquête de décembre 1810. Censure, répression et réorganisation du livre sous le Premier Empire, Paris, Paris-Musées, 2008, p. 169-170.

22. Le brevet est nominatif et unique : le libraire ne peut avoir plusieurs boutiques avec un seul brevet. La réitération des interdits, à ce sujet, semble néanmoins montrer une pratique différente : Gaël Mesnage, "De la rive gauche...", art. cité.

23. Arch. nat., F18 1732. Les témoins d'Adèle Mandar ne sont pas totalement inconnus, néanmoins ils sont d'une moindre envergure que ceux de son fils.

24. Arch. nat., F18 1732 : à côté du soutien du préfet de Seine-et-Oise, Eugène bénéficie égale- 
trent qu'à côté de cette inscription professionnelle, le candidat doit posséder des relations de voisinage, véritables garanties de sa réputation. En effet, Antoine Marie Denis Méquignon junior, établi rue de la Harpe ${ }^{\circ} 115$, fait signer son certificat de bonne vie et mours par des commerçants de sa rue : un marchand de draps, un marchand de vin et un boulanger, tous situés exactement à côté ou en face de chez lui ${ }^{25}$.

Cependant, les logiques spatiales et professionnelles se recoupent et les signataires des certificats de moralité sont parfois des gens du livre à proximité. Les listes de témoins des Belin ${ }^{26}$ sont éloquentes. Mère et fils font témoigner des confrères : le certificat d'Adèle Belin-Mandar est signé par deux libraires : Igonette et Lagny. Son fils propose comme témoins d'une part, en 1847 pour son brevet de libraire, deux confrères : Guillemot et Gennequin aîné et d'autre part, en 1851 pour sa demande de brevet d'imprimeur : Louis Stanislas Prioux, marchand de papier, et l'imprimeur Auguste Alexis Pillet aîné. Tous ces témoins sont extrêmement proches des postulants et situés exactement dans le même pâté de maisons, le village Saint-André-des-Arts. Les deux logiques, spatiale et professionnelle, théoriquement différentes et propres à chaque certificat, se recoupent dans la pratique. Cette confusion est d'autant plus grande que certains témoins sont récurrents et apparaissent comme signataires des deux certificats soulignant ainsi une relation privilégiée, ici, entre Belin-Mandar et Igonette, et entre Belin fils et Pillet aîné. L'abolition de la distinction entre les deux certificats, de moralité et de capacité, révèle tout à la fois l'importance du voisinage dans la reconnaissance professionnelle et la richesse des liens tissés entre confrères.

Pourtant, dans ces certificats, au contraire des actes d'état civil, jamais la nature du lien entre les témoins et le candidat n'est mentionnée. Les signataires appuyant le futur libraire ont-ils véritablement le choix ou imposent-ils leurs conditions, et pourquoi pas des conditions financières? Il est délicat de répondre catégoriquement, il semble néanmoins que ces signatures, qui s'expliquent par l'extrême concentration des libraires, impliquent un autre type de relations - non plus seulement commerciales mais situées à la limite du professionnel - organisées autour du quartier, entendu ici comme un espace d'interconnaissances et recouvrant en partie la notion de voisinage. Si cette interconnaissance contraint les signataires, elle semble être une source de la crédibilité et de la réputation publiques des postulants, considérés pourtant dans leur intimité.

C'est dans ce sens qu'il faut comprendre l'importance des enquêtes réalisées par le commissaire de police et qui sont fondées en partie sur les "on-dit" circulant dans le quartier. Le préfet de police, pour répondre à la demande du

ment, en 1851, des recommandations de M. Casenave, vice-président du Tribunal de la Seine, et du Baron de Watterville, employé au ministère de l'Intérieur.

25. Arch. nat., F18 1802.

26. Arch. nat., F18 1732. 


\section{Carte 4 : Témoins d'Adèle Belin-Mandar et de Marie Eugène Belin}

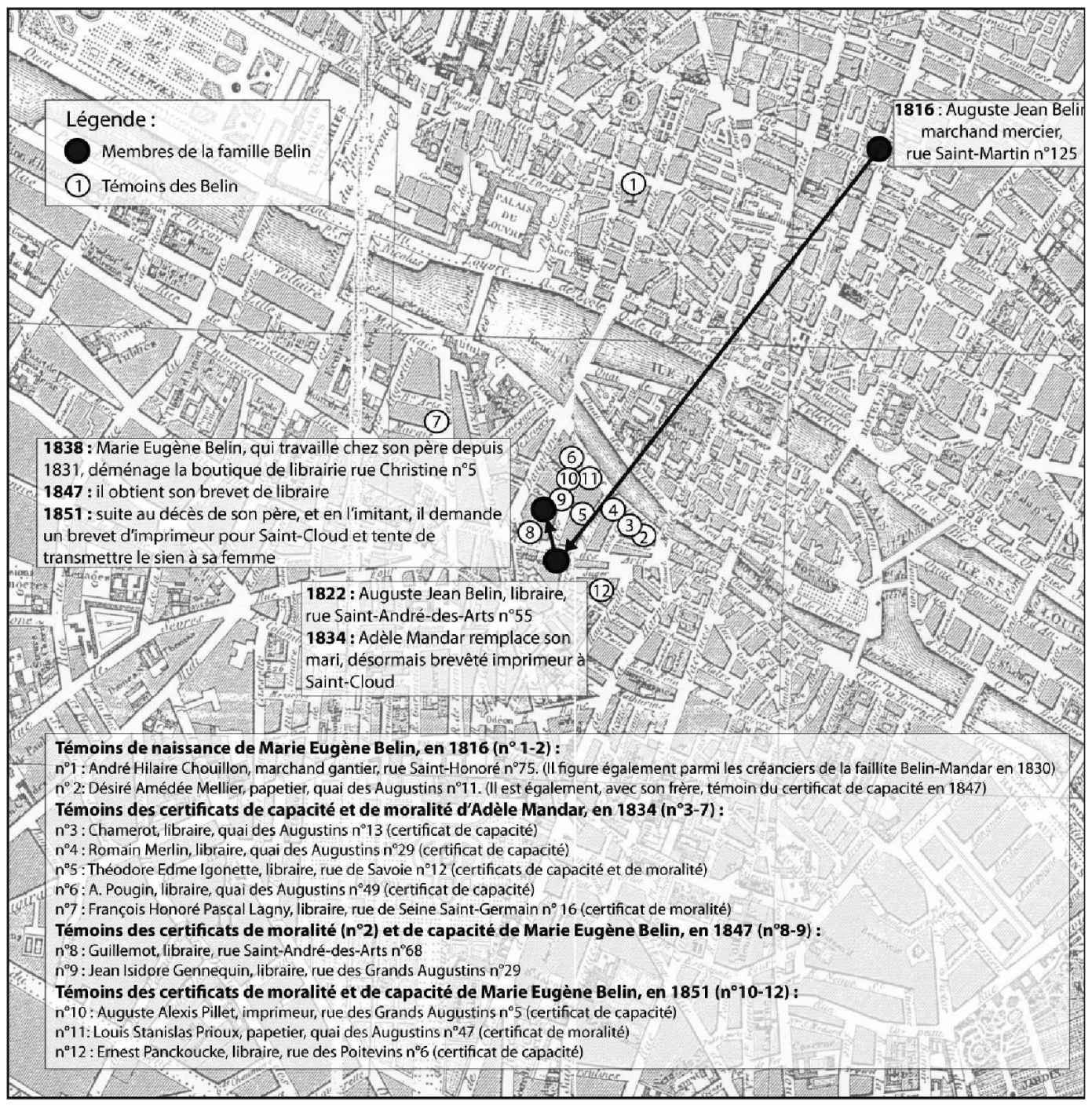




\section{Carte 5 : Quartier fondant la réputation de Boiste père}

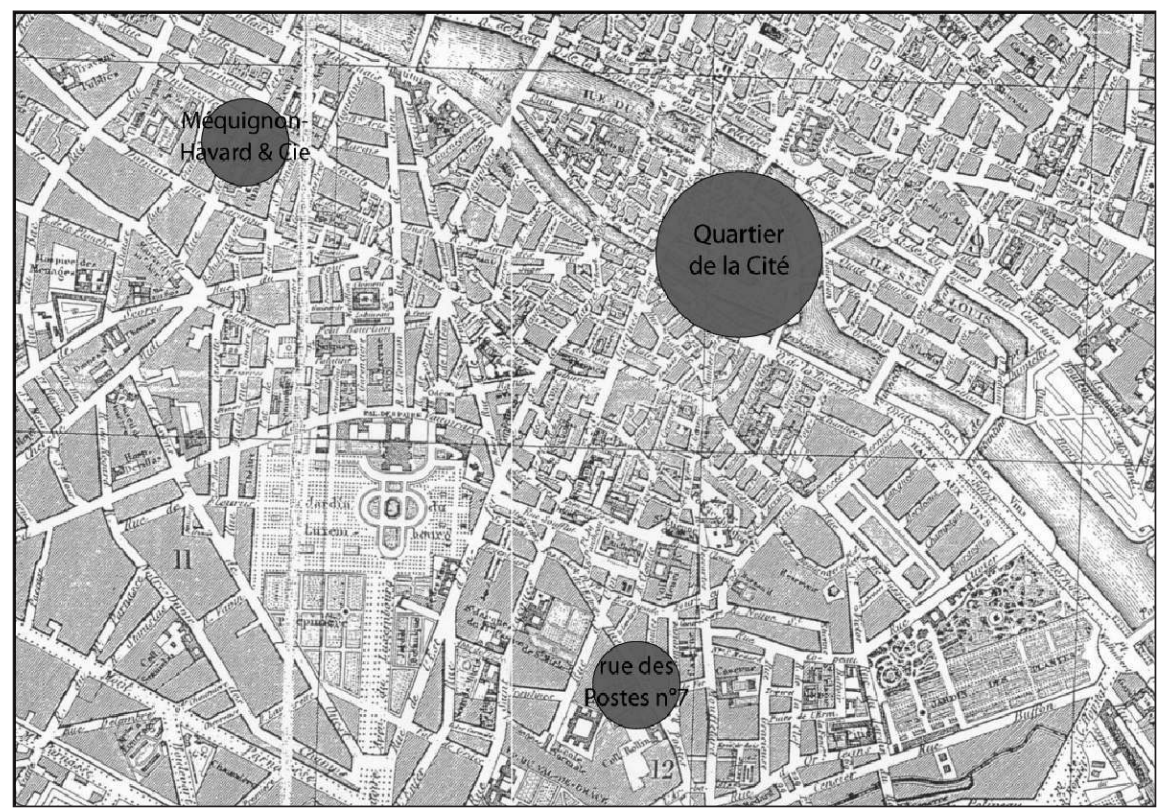

ministre secrétaire d'État, trace, en 1822, le portrait de Jean Alexandre Boiste en ces termes : «Le sieur Boiste père est âgé d'environ 45 ans, marié et père de famille. On annonce qu'après avoir été pendant plusieurs années secrétaire du commissaire de police du quartier de la Cité, il fut nommé officier de Paix, mais qu'il occupa cette place pendant très peu de temps [...]. On présente le sieur Boiste comme un homme qui s'est enrichi en faisant valoir ses fonds dans le commerce de la librairie et comme y ayant généralement la réputation d'un aigrefin en affaires et d'un usurier. [...] Quant aux opinions politiques du sieur Boiste, elles portent, dit-on, une nuance assez marquée de libéralisme. Enfin, on assure encore que lors des dernières élections, il a voté dans le sens de l'opposition. ${ }^{27}$

Il est étonnant de voir que cet ancien édile relativement aisé soit refoulé et, plus encore, balayé d'un revers de main par un collègue qui, s'appuyant sur des informations indirectes, trace un portrait particulièrement néfaste.

27. Boiste père demande, en vain, un brevet en remplacement de son débiteur Paul Claude Louis Méquignon fils aîné, en état de faillite depuis 1822. Il fonde alors une association avec la femme de son débiteur, Amélie Elisabeth Hortense Havard qui, parce qu'accusée d'être le prête-nom de Boiste père doit exercer sans autorisation, sous la raison Méquignon-Havard et Cie : Arch. nat., F18 1802; Arch. Paris, D11 U3/71, n 6416. 
Les qualifications d'usurier et d'homme libéral, empêchent Boiste d'obtenir son brevet de libraire - et donc de recouvrer ses créances. Les informations retenues par le préfet de police proviennent d'un espace relationnel assez flou mais ouvert. Le quartier qui forge la réputation du requérant est ici un espace physiquement discontinu et articulé autour de plusieurs pôles relativement éloignés : les rues liées à l'exercice de ses anciennes fonctions (le quartier de la Cité), celles liées à sa position économique (propriétaire rue des Postes $n^{\circ} 7$ ), celles (beaucoup moins lisibles) liées à l'activité financière et à ses investissements dans la maison Méquignon Havard et $\mathrm{C}^{\mathrm{ie}}$, rue des Saints Pères $\mathrm{n}^{\circ} 10$ où il a élu son nouveau domicile.

D'après le rapport policier, il est possible de penser le quartier comme un espace social plus large que celui du voisinage fondé sur l'interconnaissance, et correspondant davantage à un espace d' "intercontact" où l'information circule par tiers interposés ${ }^{28}$, pour fonder l'opinion communément partagée, sinon collectivement admise. L'exemple de Boiste souligne en outre qu'au sein de l'espace parisien des libraires, les solidarités existent mais ne sont pas mécaniques.

À côté des obligations législatives, cette logique d'inscription locale du commerce répond également aux besoins économiques imposés par la profession. La librairie est particulièrement gourmande en capitaux. Si les cadastres industriels et les déclarations de faillite montrent que le mobilier des libraires est relativement limité - quelques étagères et un comptoir dans la boutique principale, auxquels s'ajoutent tout au plus un bureau et moins d'une demidouzaine de fauteuils pour les tenants de cabinets de lecture - le coût de l'installation et les fonds en circulation sont d'une toute autre ampleur. Or, le succès n'est en aucun cas fonction des sommes investies et le risque de faillite s'accroît au rythme des méventes de ces coûteuses publications. Les liens de sociabilité de quartier, qui étaient jusque là de simples formalités administratives, sont alors activés faisant du quartier un espace de crédit. Reste à savoir si ces liens sociaux, créés par la géographie professionnelle, donnent naissance à un espace homogène et solidaire.

\section{ESPACE DE CRÉdit ET DE SOLIDARITÉ}

Avant même les bouleversements qui mettent fin à la Restauration, la librairie connaît une crise importante qui se manifeste par la multiplication des faillites. Si leur nombre culmine en 1826, puis au cours des années 1830-1831, plus qu'une crise conjoncturelle liée la situation économique londonienne ou aux événements de politique intérieure ${ }^{29}$, les faillites témoi-

28. Laurent Clavier, "Quartier” et expériences politiques...", art. cité, p. 139.

29. Henri-Jean Martin, "Le monde des éditeurs", dans Roger Chartier et Henri-Jean Martin [dir.], Histoire de l'édition française, ouv. cité, tome 3, p. 176-244. 
gnent bien plutôt d'une crise structurelle caractéristique de ce commerce. En effet, les livres restent coûteux tandis que le public ne cesse de s'élargir ${ }^{30}$. Les deux générations de faillites qui touchent la librairie correspondraient donc davantage à des recherches de solutions encore en cours d'élaboration et donnant lieu à diverses expérimentations ${ }^{31}$. Malgré l'apparente morosité, il s'agit en définitive d'une période particulièrement dynamique ${ }^{32}$. Par ailleurs, si les faillites constituent un point de rupture de la confiance entre les créanciers et leur débiteur - le règlement du contentieux devant le Tribunal de Commerce révéle de fait l'échec d'un arrangement amiable -, elles mettent surtout à l'épreuve l'entente précédemment contractée entre les deux parties, en examinant l'éventualité de son renouvellement. L'analyse des liens de crédit est donc indispensable pour comprendre les faillites.

Entre 1826 et 1848, 255 libraires et éditeurs déposent leur bilan, mais seuls quelques dossiers sont disponibles et présentent des listes de créanciers $^{33}$. Quatre catégories de créanciers, qui interviennent majoritairement dans les faillites de libraires, peuvent être distinguées : les "gens d'argent $»^{34}$, principalement situés sur la rive droite ${ }^{35}$, représentant près de $20 \%{ }^{36}$ des créanciers et un peu plus de $35 \%$ des créances faites aux libraires en faillite; les «fournisseurs " ${ }^{37}$, établis le plus souvent sur la rive gauche et constituant la moitié des créanciers mais à peine plus du tiers des sommes prêtées ${ }^{38}$; enfin, la famille - élargie aux amis - et les gens de quartier. L'inconvénient d'une telle classification réside essentiellement dans le fait que bien souvent les trois derniers groupes énoncés se recoupent dans le cas de la librairie, relativement fermée socialement et concentrée géographiquement. Seule une analyse à l'échelle des individus permet de saisir la temporalité et la spatialité des liens économiques que tissent au cours de leur activité ces libraires en faillite.

Le premier "cercle de créanciers " ${ }^{39}$ est sollicité dès l'établissement des

30. Jusqu’à la «révolution Charpentier», en 1838, le roman est à 7,50 francs le volume.

31. Ces innovations conduisent d'une part, à réduire le format pour abaisser le prix du livre, et d'autre part, à publier les romans en livraisons.

32. Jean-Clément Martin, «Le commerçant, la faillite et l'historien", Annales ESC, vol. 35, n 6, 1980, p. 1251-1268.

33. Parmi les 255 faillites ouvertes entre 1826 et 1848 inscrites sur les registres (D10 U3), seuls 52 dossiers sont conservés (D11 U3). Ils couvrent la période 1830-1848, la plupart concernant les années 1830-1831 (41 dossiers). Sur 36 de ces dossiers de faillites analysés ici, deux ne présentent pas de liste de créanciers.

34. Les véritables banquiers sont encore rares et nous entendons par ce terme : orfevres et bijoutiers, rentiers et propriétaires; Michel Lescure et Alain Plessis [dir.], Banques locales et régionales au XIXe siècle, Paris, Albin Michel, 1999.

35. 176 sur 250 «banquiers" parisiens identifiés $(70 \%)$ - l'identification de tels personnages est rendue d'autant plus complexe que certains gens du livre-escompteurs, à l'instar de Pourrat frères ou de Fantin, sont, au gré des différentes faillites, tour à tour désignés comme banquiers ou imprimeurs.

36. 313 sur 1620 créanciers.

37. Ensemble des acteurs intéressés à la production ou à la vente de livres comprenant libraires et imprimeurs, papetiers et brocheurs, ou encore hommes de lettres et employés de magasin.

38. 838 des 1648 créanciers ( $51 \%$ ), prêtent ensemble près de 2650000 sur les 8180000 francs de créances de nos 34 faillis $(32 \%)$.

39. Laurence Fontaine, L'économie morale. Pauvreté, crédit et confiance dans l'Europe préindustrielle, Paris, Gallimard, 2008. 
libraires. L'achat du fonds de commerce qui s'élève généralement à plus de 150000 francs est largement financé par la famille. Les dots des femmes mais également le soutien financier des parents proches facilitent l'entrée dans le métier. La présence des épouses, qui figurent très fréquemment au passif de la faillite de leur mari au titre de leurs reprises matrimoniales, laisse entrevoir une stratégie de protection : soit que le couple préserve les biens domestiques nécessaires à sa survie, à l'instar de David et Ève Séchard ${ }^{40}$; soit que le failli choisisse d'augmenter le nombre de créanciers à son profit ${ }^{41}$. En tout état de cause, la faillite révèle la participation financière initiale des épouses dans l'entreprise du mari : ainsi, lorsque Charles Denn achète le fonds de commerce de Louis Depélafol à 195000 francs en 1822, il ne paie comptant que 33000 francs, grâce à la dot de sa femme ${ }^{42}$. Mais, le capital dotal varie d'une femme à l'autre, selon leur statut social : tandis que Marie Cahan apporte près 10000 francs à Paul Ledoux ${ }^{43}$, Martin Bossange, qui fait faillite la même année, en 1830, en reçoit 70000 de $\mathrm{M}^{\text {elle }}$ Volland ${ }^{44}$. À la même époque, sinon aux moments de "coups durs", les parents consanguins ou alliés des libraires proposent des aides financières plus conséquentes. Amélie Havard parvient à reprendre en 1824 le fonds de son mari, tombé en faillite, grâce aux 60000 francs que lui prête sa mère. De même, Auguste Jean Belin, lorsqu'il dépose son bilan en 1830, doit à ses "oncles " ${ }^{45}$, respectivement plus de 97000 et 39000 francs; près de 60000 francs à sa mère et son frère; tandis que son associé Devaux doit à son fils plus de 30000 francs; soit ensemble plus de $30 \%$ des dettes déclarées ${ }^{46}$.

Une fois établis, et pour faire face aux dépenses quotidiennes de leur boutique, les libraires font appel à un deuxième cercle de créanciers. Les frais de fonctionnement suscitent en effet des dépenses considérables : à côté, des frais d'impression relativement lourds ${ }^{47}$, figurent les frais de voyage non moins importants ${ }^{48}$, auxquels s'ajoute encore l'ensemble des frais de diffusion, notamment de publicité - bien qu'ils soient encore rares en ce premier $\mathrm{XIX}^{\mathrm{e}}$ siècle $^{49}$. Pour couvrir ces dépenses, les libraires activent d'autres réseaux

40. Honoré de Balzac, Illusion perdues..., ouv. cité.

41. Le concordat n'est accepté qu’à la double majorité : $50 \%$ des créanciers et $75 \%$ des créances.

42. Arch. Paris, D11 U3/72, n⿳0 6432.

43. Arch. Paris, D11 U3/81, nº 6719.

44. Arch. Paris, D11 U3/79, n 6666.

45. Arch. nat., F18 1732 : Le terme d'«oncle», mentionné dans l'acte de naissance d'Eugène BelinMandar, est étonnant. Ces derniers jouent pourtant un rôle primordial, à l'instar de celui des parrains, dans la carrière d'Auguste Belin : Chouillon favorise son installation comme mercier à quelques rues de lui; Mellier est à l'origine de son double mouvement géographique et professionnel (carte 4).

46. Arch. Paris, D11 U3/75, nº 6553; Arch. nat., F18 1732.

47. Arch. Paris, D11 U3/371, nº 6416 : la publication de 1000 exemplaires en 35 volumes in-12 de la traduction française des Pères de l'Église par l'abbé Guillon, vaut l'investissement de 50000 francs à Amélie Havard.

48. Arch. Paris, D11 U3/372, n ${ }^{\circ} 6432$ : Le bilan de Charles Denn mentionne près de 40000 francs de frais de voyage en huit années d'activité, soit près de 400 francs par mois, soit autant que ses frais de commis, exploitation, loyers, impositions.

49. Quatre courtiers d'annonces, dits ensuite "agents de publicités», apparaissent dans huit faillites de libraires après 1847 (Sylvain Dusseris, la société Lafitte Bullier et Cie, Richard et fils, Defos et Cie). 
de crédit, à savoir les fournisseurs et les gens de quartier, souvent confondus.

Tableau 2 : Répartition des créances, faites aux libraires
par leurs fournisseurs, selon leur localisation

\begin{tabular}{|c|c|c|c|c|}
\hline \multirow{2}{*}{$\begin{array}{l}\text { Lieu de résidence des } \\
\text { créanciers }\end{array}$} & \multicolumn{2}{|c|}{ Créanciers } & \multicolumn{2}{|c|}{ Somme des créances } \\
\hline & Effectifs & $\%$ & en francs & $\%$ \\
\hline \multicolumn{5}{|c|}{ Arrondissements parisiens } \\
\hline$I$ & 19 & $2,27 \%$ & 117611,43 & $4,43 \%$ \\
\hline II & 69 & $8,23 \%$ & 108976,64 & $4,11 \%$ \\
\hline III & 33 & $3,94 \%$ & 66704,55 & $2,51 \%$ \\
\hline IV & 35 & $4,18 \%$ & 68446,69 & $2,58 \%$ \\
\hline $\mathbf{v}$ & 4 & $0,48 \%$ & 4813,29 & $0,18 \%$ \\
\hline VI & 11 & $1,31 \%$ & 92580,70 & $3,49 \%$ \\
\hline VII & 5 & $0,60 \%$ & 4896,60 & $0,18 \%$ \\
\hline VIII & 1 & $0,12 \%$ & 1060,00 & $0,04 \%$ \\
\hline IX & 5 & $0,60 \%$ & 49235,85 & $1,86 \%$ \\
\hline$x$ & 180 & $21,48 \%$ & 961560,56 & $36,24 \%$ \\
\hline $\mathbf{X I}$ & 376 & $44,87 \%$ & 874306,44 & $32,95 \%$ \\
\hline XII & 53 & $6,32 \%$ & 203385,38 & $7,67 \%$ \\
\hline Paris & 791 & $94,39 \%$ & 2553578,13 & $96,24 \%$ \\
\hline \multicolumn{5}{|c|}{ Communes limitrophes } \\
\hline Belleville & 2 & $0,24 \%$ & 332,75 & $0,01 \%$ \\
\hline Montmartre & 2 & $0,24 \%$ & 975,50 & $0,04 \%$ \\
\hline \multicolumn{5}{|c|}{ Ile de France hors Paris et ses communes limitrophes } \\
\hline Seine et Oise & 6 & $0,72 \%$ & 23886,04 & $0,90 \%$ \\
\hline Seine et Marne & 5 & $0,60 \%$ & 9865,85 & $0,37 \%$ \\
\hline \multicolumn{5}{|c|}{ France hors Ile de France } \\
\hline France hors IdF & 31 & $3,70 \%$ & 64476,83 & $2,43 \%$ \\
\hline \multicolumn{5}{|c|}{ Indéterminé } \\
\hline Indéterminé & 1 & $0,12 \%$ & 190,68 & $0,01 \%$ \\
\hline Total & 838 & $100,00 \%$ & 2653305,78 & $100,00 \%$ \\
\hline
\end{tabular}

Certes, les faillites ne livrent qu'un discours indirect et incomplet sur l'espace des sociabilités, qu'un reflet imparfait des relations - de solidarité ou de civilité - entretenues et pensées par les acteurs transformant ainsi l'espace physique en espace socialement investi ${ }^{50}$. Néanmoins, et bien qu'ils ne constituent que la partie émergée de l'iceberg - le geste de demande de crédit -, les liens de crédit, rendus visibles par la cessation de paiement, livrent

50. Pilar González Bernaldo de Quirós, "Sociabilité urbaine», Hypothèses 2008. Travaux de l'école doctorale d'histoire de l'Université Paris 1 Panthéon-Sorbonne, Paris, Publications de la Sorbonne, 2009, p. 295-303. 
une géographie de l'endettement permettant de comprendre cet espace de crédit fortement localisé. Cette géographie centrée sur l'espace vécu de chacun - réseau de type égocentré - présente-t-elle un espace collectif cohérent? La proximité permet-elle de choisir ou non de prêter à son voisin et confrère? Et plus généralement, ces réseaux financiers de quartier relèvent-ils d'une solidarité à toute épreuve?

Parmi les 37 créanciers de Charles Denn ${ }^{51}$, installé dans le quartier de l'École de Médecine, vingt se situent dans les $\mathrm{X}^{\mathrm{e}}$ et $\mathrm{XI}^{\mathrm{e}}$ arrondissements, dont cinq dans le quartier de la Monnaie et douze dans son propre quartier. Sur les cinq créanciers du quartier de la Monnaie, deux relèvent de la «famille»: on distingue $\mathrm{M}^{\mathrm{me}}$ Denn, son épouse, habitant rue des Marais Saint-Germain $\mathrm{n}^{\circ} 4$, et une voisine du même immeuble, $\mathrm{M}^{\mathrm{me}} \mathrm{Mir}$, qui apportent ensemble environ 60000 francs au libraire en faillite, soit plus de la moitié des sommes prêtées à Charles Denn. Par ailleurs, les créanciers du quartier de l'École de médecine sont tous situés dans les rues adjacentes des établissements successifs du libraire. Le quartier n'est plus seulement le support de la réputation politique, mais également comptable, des acteurs et la proximité apparaît comme une véritable caution protectrice pour chacune des parties, débiteurs et créanciers. En effet, le lien de crédit n’est pas simplement une relation de solidarité - $\mathrm{M}^{\mathrm{me}}$ Mir ne subit peut-être pas autant sa relation de voisinage qu'elle ne choisit de placer son argent utilement - mais également un lien de dépendance mutuelle - non réciproque - entre débiteur et créancier. Tandis que le premier espère obtenir les conditions financières les plus avantageuses, soit le plus faible taux d'intérêt possible, le créancier mise avant tout sur la solvabilité de son "client» espérant de celui-ci qu'il recouvre promptement, voire fasse fructifier, ses investissements. Or, cette relation se noue sur une sorte de confiance qui repose sur la réputation, ou l'insertion sociale, de chacun. Le quartier est donc ici un espace de crédibilité, dans tous les sens du terme ${ }^{52}$.

Les créanciers vérifiés par le syndic de faillite de Charles Denn qui demeurent en dehors de ces deux quartiers sont majoritairement des fournisseurs $(90 \%)$ de proximité $(80 \%)$ ou, parfois, de province. Hors du quartier la réputation se fonde sur l'insertion au sein de la communauté marchande ou professionnelle ${ }^{53}$.

En effet, à côté des "solidarités de voisinage» qu'illustre le prêt octroyé par $\mathrm{M}^{\mathrm{me}}$ Mir à Charles Denn, les créanciers de quartier comptent de manière

51. Arch. Paris, D11 U3/72; dossier $n^{\circ} 6432$.

52. Sur les boulangers ou les marchands de vin comme créanciers : Bernadette Angleraud, «Le pain quotidien : la boutique boulangère au cœur des sociabilités de quartier au XIXe siècle», dans Natacha Coquery [dir.], La boutique et la ville. Commerces, commerçants, espaces et clientèles, XVI'-XX' siècle. Actes du colloque des 2, 3 et 4 décembre 1999 organisé par l'université François Rabelais, Tours, Tours, Publication de l'Université de Tours, 2000, p. 368-380; Laurent Clavier, "Quartier" et expériences politiques...", art. cité.

53. Jean-Clément Martin, «Le commerçant, la faillite... », art. cité; Pierre-Cyrille Hautcœur, «Pour une approche quantitative des faillites", Histoire et Mesure, 2008, vol. 23, nº 1, p. 3-17. 
Carte 6 : Réseaux de créanciers de Denn et Depélafol

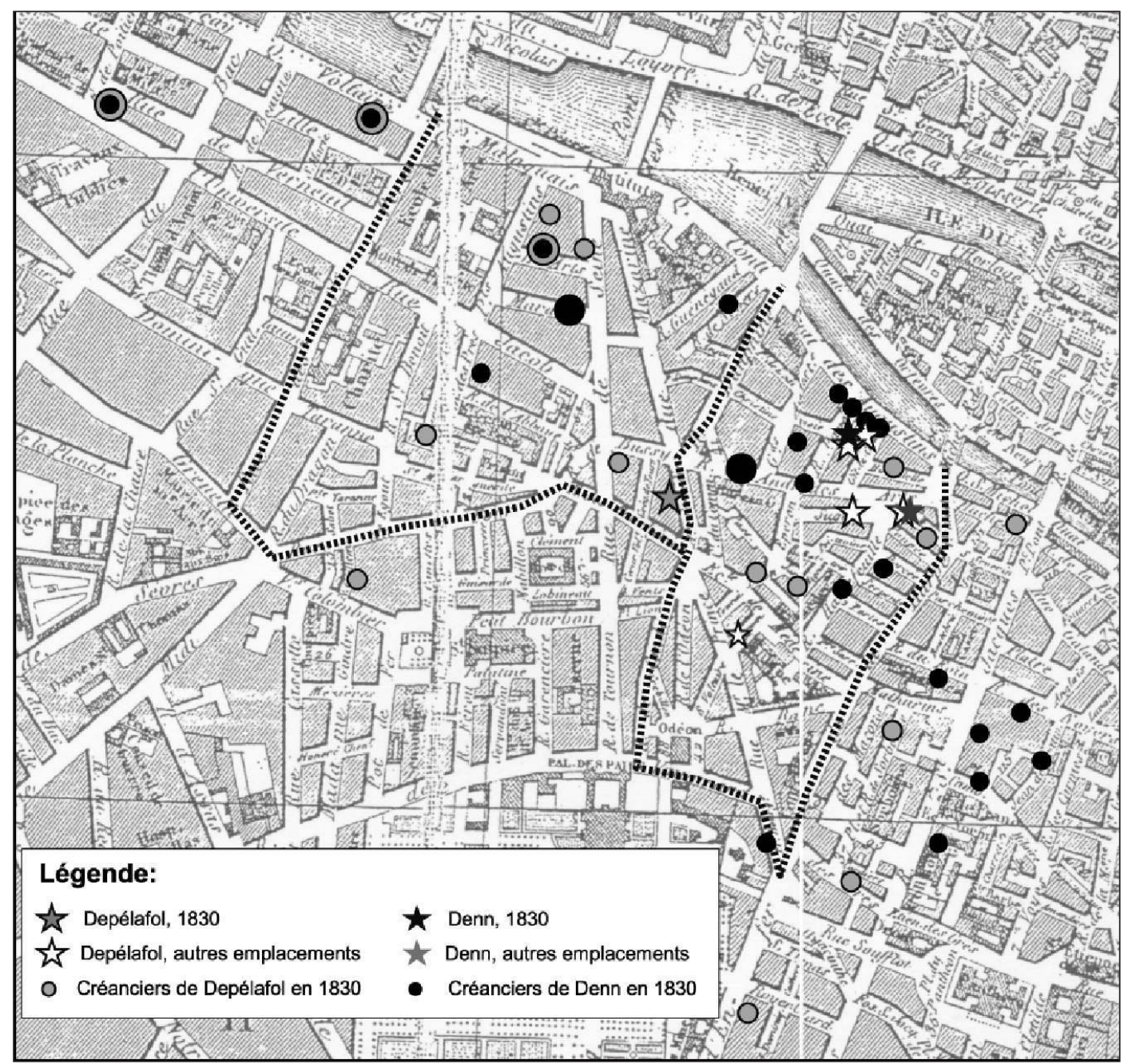


générale un grand nombre de fournisseurs. Imprimeurs et papetiers prêtent les sommes les plus élevées : chacun d'eux prête respectivement en moyenne 4000 et 4500 francs. Mais, si les libraires et éditeurs font des crédits plus modestes - environ 2500 francs en moyenne par personne -, ils restent les plus nombreux. Quelle que soit leur profession, tous fournissent les marchandises sur avance, avec billets à ordre pour preuve. Le crédit commercial est ici largement imposé par le procédé de fabrication du livre et, papetiers et imprimeurs, situés en amont de la "chaîne du livre» sont plus fréquemment contraints de se plier aux désirs de leurs clients. Pourtant la récurrence des noms de certains d'entre eux dans diverses faillites pose question : sont-ils victimes de leur succès - à tendance monopolistique -, à l'instar des papetiers Auzou ou Montgolfier, des libraires Panckoucke, Levavasseur, etc.; ou bien représentent-ils des Gens du livre qui s'orientent vers une activité financière parallèle ?5 $^{54}$

À côté du cas de Boiste déjà évoqué, qui reste extérieur au commerce qu'il finance - tout à la fois officier de paix et d'opinion politique libérale, il se place ainsi en dehors du secteur professionnel et économique de la librairie dans laquelle il investit, celle-ci étant d'obédience catholique et monarchique -, certains «marchands de livres» se reconvertissent à l'escompte de la librairie, à l'instar du Chaboisseau de Balzac ${ }^{55}$. Si l'écrivain, particulièrement bien placé pour évoquer ces "usuriers", dénonçait cette activité comme particulièrement lucrative pour ses héros aux doigts agiles, il est difficile d'évaluer les bénéfices que ces libraires tirent de telles opérations : il faut croire qu'ils ne spéculent qu'acculés aux difficultés financières, expliquant par là-même le fait que le développement de cette double activité ait lieu en pleine «crise du livre", dans les années 1820-1830. Ainsi, Adrien Thoisnier Desplaces, qui obtient son brevet de libraire en $1822^{56}$, tente de faire face à l'incendie de sa succursale new-yorkaise, tenue par son frère, en investissant davantage : dès 1826, il ouvre une deuxième boutique parisienne rue Vivienne, à côté de celle de la rive gauche, et diversifie ses activités, se faisant escompteur de librairie ${ }^{57}$. Il en est de même pour Louis Mame et René Delaunay qui, face à leurs difficultés et à celles de leurs confrères, créent, à côté de leur librairie, une société pour mettre des billets en circulation ${ }^{58}$.

L'activité de ces libraires-escompteurs met au jour l'espace local des réseaux de crédit des libraires parisiens et permet, à partir de l'analyse d'un réseau centré sur Louis Mame et René Delaunay ainsi que sur Armand Depé-

54. La spéculation des libraires s'explique sans doute par la prise de risque et l'incertitude des affaires de ce commerce; Laurence Fontaine, L'économie morale..., ouv. cité, p. 301.

55. Le chaboisseau est un cétacé considéré comme le "crapaud des mers", c'est dire toute l'estime que Balzac a pour les libraires.

56. Arch. Paris, D11 U3/75, no 6545.

57. Arch. Paris, D11 U3/75, n 6545. 155.

58. Arch. Paris, D11 U3/72, n 6431; Nicole Felkay, Balzac et ses éditeurs..., ouv. cité, p. 139- 
lafol, de mieux comprendre la structure sociale de ce secteur dans les années 1830. Suite à la mort de son associé en 1816, Depélafol, libraire de 1812 à 1822 , se convertit à l'escompte de la librairie ${ }^{59}$ et transmet son brevet ainsi que son fonds de commerce à Charles Denn. Mais, faute de fonds suffisants, c'est Depélafol qui poursuit véritablement les affaires, Denn parcourant la France pour diffuser ses publications ${ }^{60}$. Depélafol s'engage alors auprès de plusieurs librairies, rachetant les billets à ordre de chacune d'elles, au point d'être jugé en partie responsable des faillites de quelques unes. Le syndic de la faillite d'Armand Depélafol signale qu' «avec Emler frères par exemple, il s'engagea jusqu'à la concurrence d'une somme de 130000 francs et fut obligé de donner quittance moyennant 90000 francs qu'il reçut en ouvrages de librairies $[\ldots] \aleph^{61}$. Dans la maison Méquignon-Havard, il détient également mille exemplaires de la traduction des Pères de l'Église par l'abbé Guillon, d'un montant de 50000 francs, en nantissement.

Non seulement parce que les valeurs sont très majoritairement prêtées par le biais de billets à ordre, dont le jeu de signatures impliquent des relations financières complexes, mais également parce que Mame-Delaunay comme Depélafol représentent des intermédiaires de crédit faisant lien, il résulte de cette situation un ensemble de relations triangulaires entre les différents acteurs de la librairie. Ces relations sont compréhensibles en termes d'analyse de réseau, comme le suggère le graphe page suivante.

Si la technique des graphes présente des limites, qu'il faut toujours avoir à l'esprit ${ }^{62}$, elle permet, une fois les données recontextualisées, de donner une visibilité à la structure sociale et spatiale de la géographie parisienne du livre.

En effet, si chaque failli détient son lot de prêteurs particuliers, souvent issus de leur clan, le graphe souligne surtout l'importance des personnages qui interviennent dans différentes faillites. On peut distinguer d'une part des confrères situés dans le même quartier que les faillis - tels Fantin, Decourchant, Pourrat frères, etc. - plus ou moins incontournables et d'autre part, des banquiers localisés sur la rive droite, à distance de leurs débiteurs. Ici, comme Mame et Delaunay-Vallée, Depélafol finance ses escomptes de librairie en sollicitant le soutien des maisons de banque comme Genella, FrévilleLevingt et Cie, Ador-Vernier et Cie, etc. Mais ces libraires-escompteurs sont

59. Arch. Paris, D11 U3/71, no 6426 : c'est d'ailleurs au titre de cette conversion dans l'escompte que le syndic de la faillite tente, en 1830, de le disculper, puisque seuls les commerçants peuvent être soumis à cette procédure collective.

60. Arch. Paris, D11 U3/72, n ${ }^{\circ} 6432$.

61. Arch. Paris, D11 U3/71, nº 6426.

62. Claire Lemercier, "Analyse de réseaux et histoire", Revue d'histoire moderne et contemporaine, $2005, n^{\circ} 52-2$, p. 91 . Ajoutons qu'il ne s'agit ici que des liens de crédit et non pas de l'ensemble des liens sociaux, de l'ensemble du "capital relationnel » propre à chaque individu. Par ailleurs, aucune visibilité n'est donnée aux relations entre différents faillis ayant recours aux mêmes créanciers, ni des divers créanciers prêtant aux mêmes libraires. Enfin, les points symbolisant les individus ne distinguent pas explicitement le fait que certains faillis sont également créanciers, et réciproquement. Le graphe présente donc surtout ici les intermédiaires du crédit. 
Figure 1 : Réseaux de crédit autour de Armand Louis Depélafol ${ }^{63}$

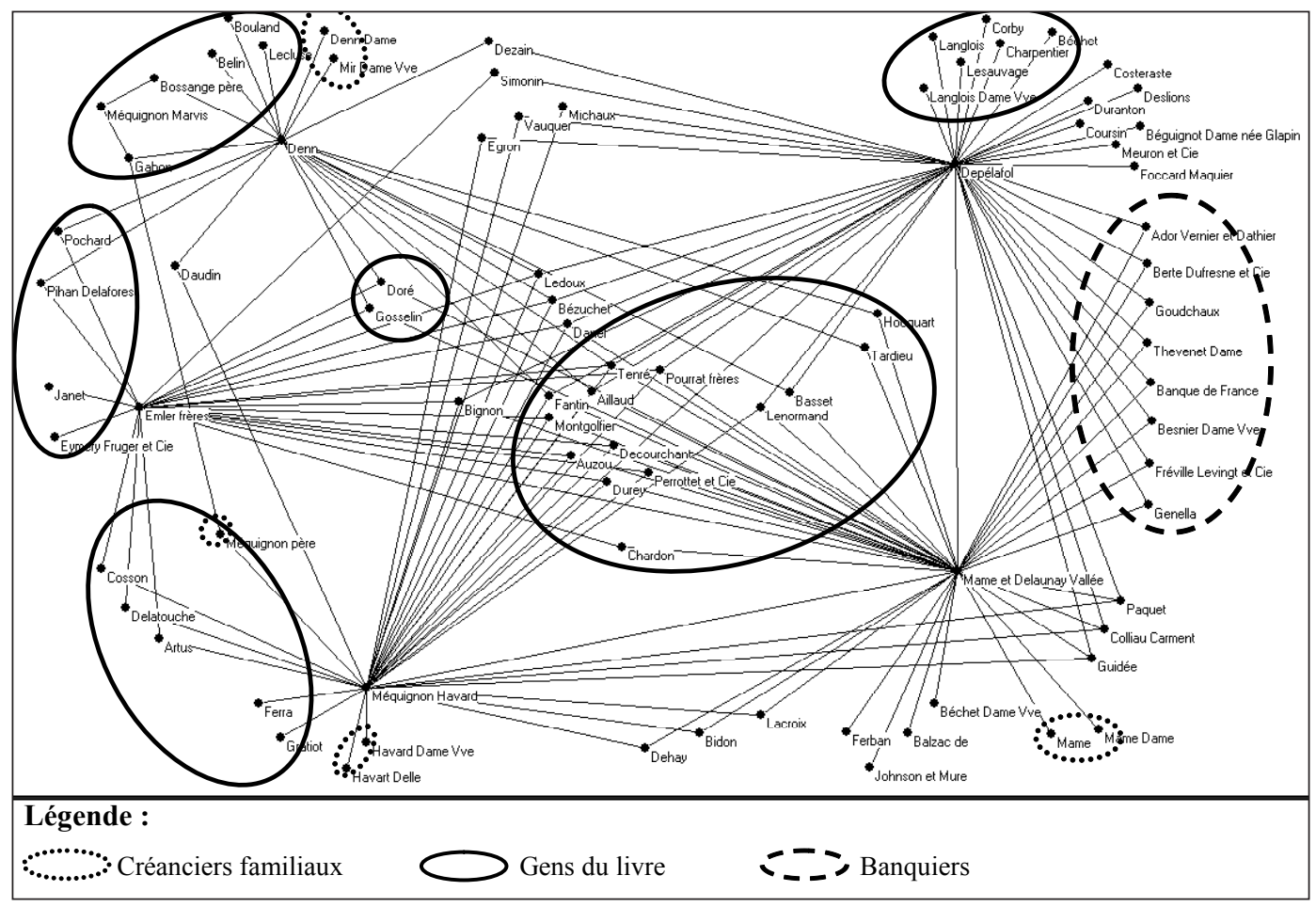

les seuls à posséder des réseaux aussi élargis géographiquement et professionnellement. Cette exception souligne paradoxalement l'importance des relations de quartier sur lesquelles se fonde le fonctionnement de la librairie. Autrement dit, il semble que la librairie s'organise comme une économie locale s'appuyant sur un système de «micro-crédit» donnant toute sa place à l'espace du quartier, qui est loin d'être replié sur lui même. Certains libraires, aux activités diversifiées ou économiquement florissantes, accèdent en effet à d'autres intermédiaires à l'échelle de la ville, voire au-delà. L'espace de la librairie est donc, au cours de la monarchie de Juillet, hiérarchisé selon l'aire de recours financier mobilisable, cette structure tout à la fois auréolaire et réticulaire pouvant susciter, on l'imagine aisément, de nouvelles relations cette fois conflictuelles.

63. En dehors des membres de la famille, nous n'avons sélectionné que les créanciers récurrents au sein des faillites de Emler frères, Havard-Méquignon, Depélafol, Mame et Delaunay-Vallée et Denn. 
La discontinuité physique qui marque la géographie parisienne du livre révèle un espace social fortement polarisé. La librairie parisienne s'inscrit au sein de réseaux de confiance locaux, symboliques et matériels, la réputation des libraires ainsi que la source des financements indispensables en cas de faillite se fondant à l'échelle du quartier. Cet espace de crédibilité s'élargit au gré de la réussite économique et de la longévité de l'entreprise. Tandis que les petits libraires restés dans l'ombre n'ont d'assise qu'à l'échelle du quartier, les grands barons de l'édition et les libraires-escompteurs peuvent se prévaloir d'alliances extérieures, à la fois hors du quartier et de la profession, ce qui leur permet d'accroître leur influence au sein de l'espace local. L'espace de crédit révèle donc une structure sociale et professionnelle hiérarchisée.

Au cours du second XIX ${ }^{e}$ siècle, avec l'évolution des structures économiques - qu'il s'agisse de l'apparition de nouveaux intermédiaires de crédit, comme les banquiers, ou des transformations des stratégies éditoriales -, la géographie parisienne du livre se dilate et la cohérence de l'espace local investi par les libraires éclate. La rupture apparait vers 1855, comme un contrecoup aux révolutions de 1848 : la disparition des libraires-escompteurs signe la fin d'une période de modernité spécifique à la librairie de la monarchie de Juillet, tout à la fois héritière de l'Ancien Régime et créatrice d'un espace professionnel inédit.

Viera Rebolledo-Dhuin est doctorante en histoire à l'Université de Versailles Saint-Quentin-en-Yvelines 